\title{
The Effectiveness of Three Regions in Mitochondrial Genome for Aphid DNA Barcoding: A Case in Lachininae
}

\author{
Rui Chen ${ }^{1,2}$, Li-Yun Jiang ${ }^{1 *}$, Ge-Xia Qiao ${ }^{1 *}$ \\ 1 Key Laboratory of Zoological Systematics and Evolution, Institute of Zoology, Chinese Academy of Sciences, Chaoyang District, Beijing, People's Republic of China,
} 2 Graduate University of Chinese Academy of Sciences, Shijingshan District, Beijing, People's Republic of China

\begin{abstract}
Background: The mitochondrial gene $\mathrm{CO}$ has been widely used by taxonomists as a standard DNA barcode sequence for the identification of many animal species. However, the $\mathrm{COI}$ region is of limited use for identifying certain species and is not efficiently amplified by PCR in all animal taxa. To evaluate the utility of COI as a DNA barcode and to identify other barcode genes, we chose the aphid subfamily Lachninae (Hemiptera: Aphididae) as the focus of our study. We compared the results obtained using $\mathrm{COI}$ with two other mitochondrial genes, COll and Cytb. In addition, we propose a new method to improve the efficiency of species identification using DNA barcoding.

Methodology/Principal Findings: Three mitochondrial genes (COI, COII and Cytb) were sequenced and were used in the identification of over 80 species of Lachninae. The $\mathrm{COI}$ and COIl genes demonstrated a greater PCR amplification efficiency than Cytb. Species identification using COIl sequences had a higher frequency of success (96.9\% in "best match" and $90.8 \%$ in "best close match") and yielded lower intra- and higher interspecific genetic divergence values than the other two markers. The use of "tag barcodes" is a new approach that involves attaching a species-specific tag to the standard DNA barcode. With this method, the "barcoding overlap" can be nearly eliminated. As a result, we were able to increase the identification success rate from $83.9 \%$ to $95.2 \%$ by using $\mathrm{COI}$ and the "best close match" technique.

Conclusions/Significance: A COII-based identification system should be more effective in identifying lachnine species than $\mathrm{COl}$ or Cytb. However, the Cytb gene is an effective marker for the study of aphid population genetics due to its high sequence diversity. Furthermore, the use of "tag barcodes" can improve the accuracy of DNA barcoding identification by reducing or removing the overlap between intra- and inter-specific genetic divergence values.
\end{abstract}

Citation: Chen R, Jiang L-Y, Qiao G-X (2012) The Effectiveness of Three Regions in Mitochondrial Genome for Aphid DNA Barcoding: A Case in Lachininae. PLoS ONE 7(10): e46190. doi:10.1371/journal.pone.0046190

Editor: Valdur Saks, Université Joseph Fourier, France

Received May 30, 2012; Accepted August 29, 2012; Published October 3, 2012

Copyright: (c) 2012 Chen et al. This is an open-access article distributed under the terms of the Creative Commons Attribution License, which permits unrestricted use, distribution, and reproduction in any medium, provided the original author and source are credited.

Funding: This work was funded by the National Natural Sciences Foundation of China (No. 30830017), National Science Funds for Distinguished Young Scientists (No. 31025024), National Science Fund for Fostering Talents in Basic Research (No. J0930004), and the Ministry of Science and Technology of the People's Republic of China (MOST Grant No. 2011FY120200). The funders had no role in study design, data collection and analysis, decision to publish, or preparation of the manuscript.

Competing Interests: The authors have declared that no competing interests exist.

*E-mail: jiangliyun@gmail.com (L-YJ); qiaogx@ioz.ac.cn (G-XQ)

\section{Introduction}

DNA sequences can be useful tools for the taxonomic identification of biological materials [1]. DNA barcoding has been proposed as a method to efficiently describe biodiversity. The mitochondrial gene encoding cytochrome c oxidase I (COI) has been widely used as a DNA barcode in many animal species [2]. However, numerous problems have arisen with the use of this gene as a barcode [3-6], largely due to its low level of variation among species [7-11]. These issues suggest that DNA barcode technology should be improved and that DNA barcode genes should be identified that more efficiently distinguish among species.

Aphids are a group of phloem-feeding insects belonging to the order Hemiptera, which comprises more than 4700 species [1213]. Aphids of the subfamily Lachninae [14] feed on plants in Coniferae, on some broad-leaf plants and on the roots of some weed species [15]. There are 339 known species of Lachninae distributed among 18 genera, composing 3 tribes worldwide. Lachninae are an ideal target for DNA barcoding as some genera in the subfamily feed on specific host plants, which facilitates identification at the genus level by morphological characteristics and unique host plants. However, the identification of congeneric Lachninae species is difficult due to the shortage of effective morphological characteristics available to distinguish among the large number of closely related species.

To evaluate the effectiveness of alternative mitochondrial genes as DNA barcodes and to identify useful markers that will improve the rate of species identification, we chose to focus on Lachninae as a model system. We examined three mitochondrial genes as molecular markers, COI (657 bp), COII (671 bp) and Cytb (730 bp), and we compared the utility of these genes in demonstrating sequence diversity sufficient to efficiently distinguish among species. We sequenced and analyzed 1098 sequences from 3 mitochondrial genes representing 83 species in 14 genera and the 3 tribes of Lachninae. In addition, we propose a new method to improve the DNA barcoding identification system using the optimal markers. 


\section{Results}

\section{Saturation analysis}

We tested the substitution saturation in 3 mitochondrial gene sequences using DAMBE 4.5.20. The results revealed that the sequence transitions and transversions in each gene demonstrated a linear relationship (Figure S1), thus permitting their use in DNA barcoding.

\section{Efficiency of PCR amplification}

We calculated the efficiency of PCR amplification of COI, COII and Cytb sequences for all samples and determined that the rate of success was $95.51 \%, 99.44 \%$ and $82.58 \%$, respectively, with COII demonstrating the highest probability of amplification. The COI primers had a $2.92 \%$ probability of amplifying sequences from parasitic wasps, which frequently lay their eggs in aphids. Primers for COII and Cytb genes did not show nonspecific amplification.

\section{NJ Tree structure}

The results of the overall $\mathrm{NJ}$ analysis of the COI, COII and Cytb regions of distances among samples representing 83 species are summarized in Figure 1. It should be noted that the trees presented here are intended to represent only the distance matrix and should not be interpreted as a phylogenetic hypothesis.

In the three $\mathrm{NJ}$ trees, the majority of species formed distinct clusters, except for some species such as $L$. siniquercus, $L$. tropicalis, $L$. roboris and L. yunlongensis in the genus Lachnus (Figures 1, 2) and $C$. pinea, C. atrotibialis and C. piniphila in the genus Cinara (Figures 1, $\mathrm{S} 2)$. Species in outgroups were embedded in Lachninae in the COI tree, while in the COII and Cytb trees, the ingroups formed a monophyletic group within Lachninae. In the COII and Cytb trees, both $C$. piceae and $C$. brevisaeta clustered together, respectively; but in the COI tree, both species did not form their own cluster, respectively. Due to its high intraspecific divergence, C. piniarmandicola formed a polyphyletic group in the Cytb tree. A greater number of species formed single clusters in the COII tree compared to the $C O I$ and $C y t b$ trees. In addition, we amplified 10 sequences from parasitic wasps using primers for the COI region, and these non-specific sequences did not appear in the COII and Cytb trees.

\section{Data analysis}

For the $C O I$ sequence, 658 bp was used for data analysis, among which 345 sites were conserved, 313 were variable and 264 were parsimony-informative. These sequences are heavily biased toward $\mathrm{A}$ and $\mathrm{T}$ nucleotides, averaging $40.1 \% \mathrm{~T}, 14.2 \% \mathrm{C}$, $35.5 \% \mathrm{~A}$ and $10.2 \% \mathrm{G}$. For the COII sequence, $671 \mathrm{bp}$ was used for data analysis. Of these nucleotides, 335 sites were conserved, 333 were variable and 295 were parsimony-informative. These sequences are similarly heavily biased toward $\mathrm{A}$ and $\mathrm{T}$ nucleotides, averaging $40.1 \% \mathrm{~T}, 12.5 \% \mathrm{C}, 40.0 \% \mathrm{~A}$ and $7.4 \% \mathrm{G}$. For the Cytb sequence, $730 \mathrm{bp}$ was used for data analysis. Of these nucleotides, 350 sites were conserved, 380 were variable and 327 were parsimony-informative. The average nucleotide composition for Cytb is $42.4 \% \mathrm{~T}, 12.9 \% \mathrm{C}, 35.8 \% \mathrm{~A}$ and $9.0 \% \mathrm{G}$.

The interspecific divergence of congeneric species was assessed by average inter-specific distance (K2P distance) among the species within a genus, each of which was represented by at least two species in this study (Table 1). The intra-specific divergence of congeneric species was evaluated by 3 additional parameters: the average intra-specific difference, theta $(\theta)$ and average coalescent depth $[4,16]$. The average intra-specific difference (K2P distance) was determined for all samples collected within each species that was represented by more than one individual. The theta $(\theta)$ value was calculated as the mean pairwise distance within each species with at least two representatives, which eliminates the bias associated with unequal sampling among individuals in a species. The average coalescent depth was determined to be the maximum intra-specific distance within each species that was represented by at least two individuals (Table 1).

The inter-specific divergence of con-tribe species was assessed by the average inter-specific distance (K2P distance) between all species within the genera of each tribe that were represented by at least two species (Table 2). The mean intra-specific nucleotide divergence was determined for all of the samples collected within a species that was represented by more than one individual (Table S3).

Success of similarity-based DNA identification techniques

The rate of success using the "best match" technique was $92.7 \%$ for COI, 96.9\% for COII and $93.8 \%$ for Cytb. There were 11 $(2.7 \%), 12(3.1 \%)$ and $19(6.3 \%)$ sequences of COI, COII and Cytb, respectively, that yielded ambiguous results. A total of $19 \mathrm{COI}$ sequences were misidentified (Table 3). The data set contained 1098 sequences, including 409 COI sequences, 385 COII sequences and 304 Cytb sequences. The best match for each sequence was an identical match.

To use the "best close match" technique, we determined that $95 \%$ of the intra-specific distances for the 3 markers fell between $0 \%$ and $5.54 \%$, and the latter value was used to decide whether a query had a close enough barcode match for identification. The rate of success using this method was $83.9 \%, 90.8 \%$ and $83.7 \%$ for COI, COII and Cytb, respectively (Table 3 ).

\section{Discussion}

The mitochondrial gene $C O I$ has been routinely used for species identification across the animal kingdom [2]. However, the interspecsific sequence divergence of $C O I$ and the divergence among congeneric species are highly variable depending on the different animal groups. In addition, $C O I$ is used to study lowerlevel relationships, such as those among species within a genus [17-18]. Despite its widespread use, however, the following question remains: is $\mathrm{COI}$ the most suitable DNA barcode for aphid identification?

\section{DNA barcodes may fail to identify morphologically similar species}

In our analysis, we observed that in some cases, the genetic divergence between congeneric species approached zero. These results indicated that some species groups could not be distinguished by mitochondrial gene sequences. The following analysis examines the relationships between Lachnus species with a genetic divergence of less than $1 \%$.

Lachnus siniquercus Zhang, L. tropicalis (van der Goot), L. roboris (Linnaeus) and L. yunlongensis Zhang are four related species of the genus Lachnus. These four species clustered together in the $3 \mathrm{NJ}$ trees (Figure 2).

All four of these species feed on plants of the Fagaceae family and L. yunlongensis Zhang also feeds on willow. The average divergence among the species at the three markers was $0.8 \%$ for COI (ranging from 0.5 to $2.5 \%$ ), $0.9 \%$ for $C O I I$ (ranging from 0.0 to $1.8 \%$ ) and $1.0 \%$ for Cytb (ranging from 0.0 to $2.1 \%$ ). According to our criteria, therefore, these four species are indistinguishable by the three DNA barcoding markers.

The morphological characteristics of the four species are also similar (Table 4). Differential characteristics display nearly 
A

Cinara brevisaeta (1)

-Cinara piceae (2)

- Cinara coloradensis (4)

- Cinara pinikoraiensis (4)

Cinara shinjii (1)

- Cinara anelia (1)

- Cinara largirostris (9)

- Cinara piniarmandicola etc.see figure 3

Schizolachnus (2 species) (15)

Cinara cuneomaculata (19)

- Cinara laricis (3)

- Cinara pergandei (4)

Cinara watsoni (2)

Cinara brevisaeta (1)

Cinara piceae (1)

Cinara acutirostris (1)

- Cinara pinea etc.see Figure S2

Cinara piceae (1)

- Cinara formosana (56)

- Cinara atlantica (2) Cinara pilicornis (19)

Cinara fornacula (4)

- Cinara costata (6)

- Cinara occidentalis (1)

- Cinara smaragdina (1) - Cinara pruiniviridis (2)

Cinara alba (1) Cinara longipennis (1)

- Cinara tujafilina (38)

- Cinara piceae (2)

- Lachnus quercihabitans (3)

-Maculolachnus submacula (3)

Maculolachnus sijpkensi (1)

- Longistigma liquidambarus (1)

- Longistigma caryae (1)

Macrosiphoniella yomogifoliae -Aphis glycines

Essigella knowltoni (1)

Eulachnus alticola (3)

Eulachnus nigricola (2)

Eulachnus rileyi (2)

Eulachnus piniarmandifoliae (1)

-Eulachnus drakontos (4)

Eulachnus pinitabulaeformis (2) - Pyrolachnus pyri (1) Mindarus keteleerifoliae - Trama rara (1)

-Tuberolachnus salignus (15) चे

Pyrolachnus sp. (1)

Nippolachnus piri (5)

Nippolachnus sp. (1)

Lachnus tropicalis etc.see figure 2

Lachnus longirostris (2)

-Pterochloroides persicae (19) Stomaphis pistacicola (1)

Stomaphis quercus (14)

-Stomaphis quercisucta (1)

-Stomaphis sinisalicis (3)

Stomaphis sp. (1)

Stomaphis yanonis (2)

Stomaphis betulidahuricae (2)

-Phylloxerina salicis

-Pineus armandicola $\mid$ outgoup

0.05

B

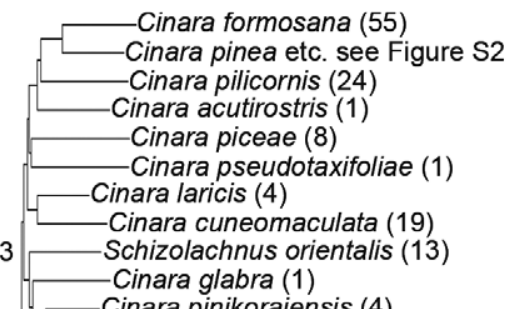

Cinara pinikoraiensis (4)

-Cinara piniarmandicola etc.see figure 3

-Cinara largirostris (9)

- Cinara etsuhoe (1)

- Cinara brevisaeta (2)

- Cinara ponderosae (1)

- Cinara costata (6)

-Cinara tujafilina (32)

-Cinara alba (1)

Cinara pruiniviridis (2)

Eulachnus nigricola (2)

-Eulachnus piniarmandifoliae (2)

-Eulachnus brevipilosus (1)

Essigella fusca (1)

Eulachnus drakontos (4)

Eulachnus rileyi (2)

Eulachnus alticola (4)

Eulachnus pinitabulaeformis (2)

Maculolachnus submacula (4)

-Stomaphis graffii (1)

-Stomaphis longirostris (1) Stomaphis pistacicola (1)

-Stomaphis japonica (1)

- Stomaphis pini (1)

-Stomaphis quercus (1)

-Stomaphis quercisucta (1)

Stomaphis sinisalicis (3)

-Stomaphis fagi (1)

Stomaphis betulidahuricae (2)

-Stomaphis sp. (1)

-Stomaphis aphananthae (1)

Stomaphis yanonis (2)

-Stomaphis aesculi (1)

- Stomaphis takahashii (1)

Nippolachnus sp. (1)

-Pyrolachnus sp. (1)

-Tuberolachnus salignus (14)

Nippolachnus piri (6)

-Protrama flavescens (1)

Protrama ranunculi (1)

Protrama radicis (1)

-Trama troglodytes (3)

-Trama maritima (2)

- Trama caudate (1)

- Trama rara (3)

- Lachnus quercihabitans (3)

Lachnus longirostris (2)

Lachnus tropicalis etc.see figure 2

- Lachnus takahashii (1)

- Longistigma liquidambarus (1)

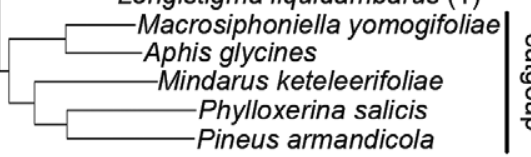

$\mid \begin{aligned} & \text { 은 } \\ & \text { 음 } \\ & \text { 듬 }\end{aligned}$

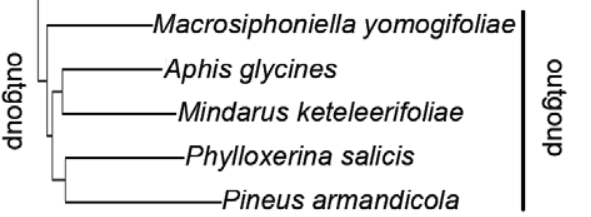

$0 . \overline{0} 1$

C Cinara tujafilina (30)

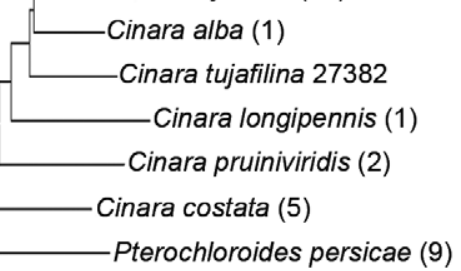

3 Lachnus quercihabitans (1)

Lachnus tropicalis etc.see figure 2

- Lachnus longirostris (2)

-Maculolachnus submacula (3)

Eulachnus nigricola (2)

Eulachnus piniarmandifoliae (2)

Eulachnus alticola (4)

-Eulachnus pinitabulaeformis (2)

-Eulachnus drakontos (4)

-Eulachnus rileyi (2)

Nippolachnus piri (5)

Nippolachnus sp. (1)

-Tuberolachnus salignus (13)

Longistigma liquidambarus (1)

-Stomaphis pistacicola (1)

Stomaphis quercisucta (1)

-Stomaphis sinisalicis (2)

Stomaphis yanonis (1)

Stomaphis betulidahuricae (1)

Cinara brevisaeta (2)

Cinara largirostris (9)

-Cinara piniarmandicola etc.see figure 3

-Cinara pinikoraiensis (4)

-Schizolachnus orientalis (10)

Cinara laricis (4)

Cinara cuneomaculata (19)

Cinara acutirostris (1)

Cinara piceae (7)

Cinara pinea etc. see Figure S2

Cinara formosana (38)

Cinara pilicornis (19)

$\overline{0.02}$

Figure 1. Basal nodes of the $\mathbf{N J}$ tree based on distances obtained using the K2P model. $\mathrm{A}$, B, and C are the COI, COIl and Cytb NJ trees, respectively. The number of identical samples is given in brackets.

doi:10.1371/journal.pone.0046190.g001

continuous variation, making identification by morphology similarly problematic.

A similar observation can be made for other Lachninae genera that are difficult to identify based on morphological characteristics. In the genus Cinara, for example, three species, C. pinea
(Zetterstedt), C. atrotibialis David \& Rajasingh and C. piniphila (Ratzeburg), clustered together in all three NJ trees (Figure S2). Similar difficulties occur in other taxa, such as birds [19], butterflies [20], fish [21] and plants [7-9]. As DNA barcodes may fail to identify morphologically similar species, it is important 


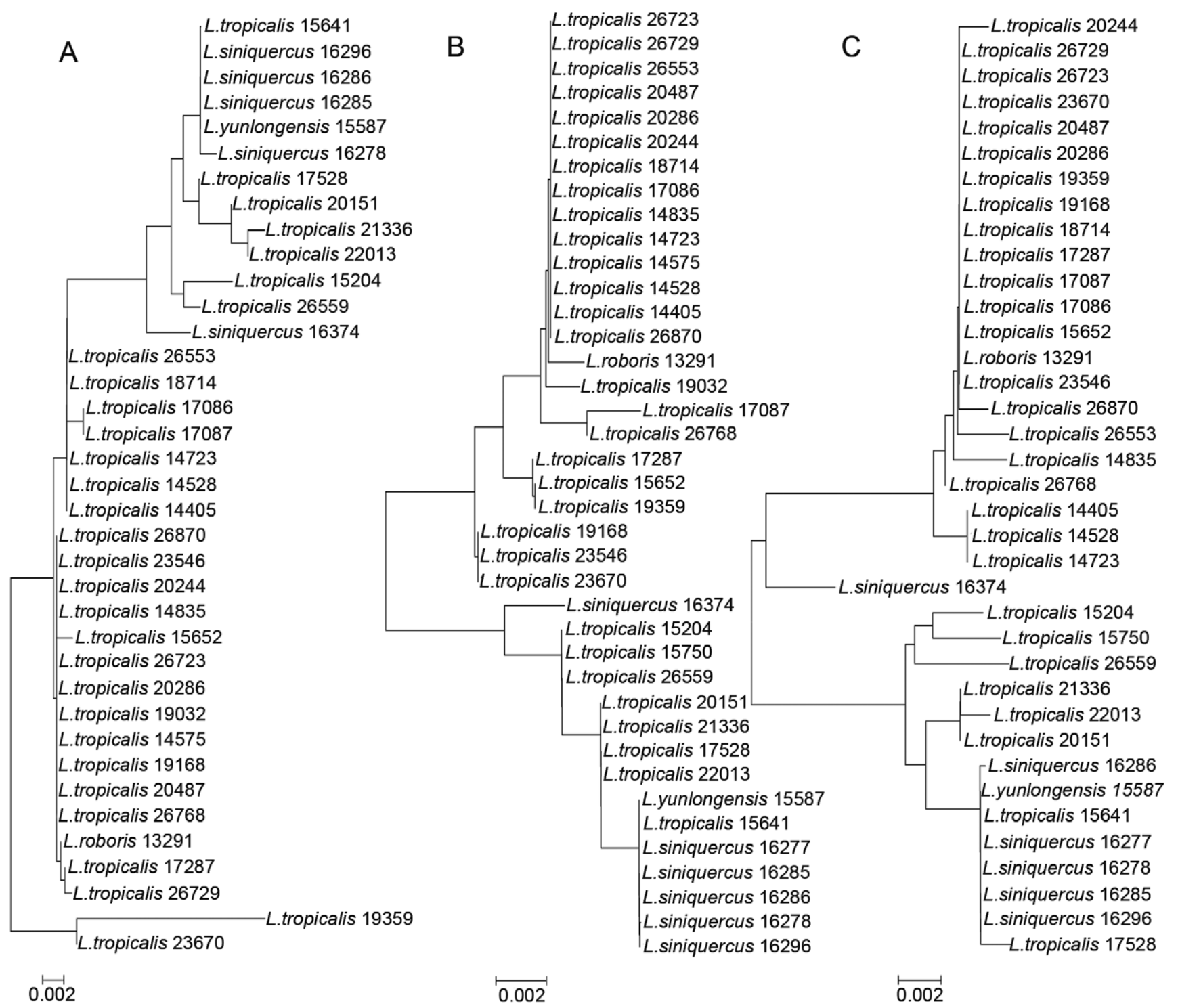

Figure 2. NJ analysis of K2P distances in Lachnus siniquercus Zhang, L. tropicalis (van der Goot), L. roboris (Linnaeus) and $L$. yunlongensis Zhang. $\mathrm{A}, \mathrm{B}$, and $\mathrm{C}$ are the $\mathrm{COI}, \mathrm{COll}$ and Cytb $\mathrm{NJ}$ trees, respectively. doi:10.1371/journal.pone.0046190.g002

for taxonomists and molecular biologists to consider the best method for classifying these species and to determine if these species are synonymous.

\section{Cytb leads to ambiguous species identification}

During our analysis of genetic divergence using Cytb, we observed intra-specific genetic divergence values of greater than $6 \%$ in some samples, especially in species from geographically distant locations, such as Cinara tujafilina (del Guercio), C. formosana (Takahashi) and C. pinea (Zetterstedt). For example, C. tujafilina (del Guercio) is widely distributed throughout China and displays a wide range of intra-specific divergence values among samples from different locations. Geographical populations show a wealth of nucleotide diversity. We observed a mean intra-specific nucleotide divergence of 0.0235 for COI, 0.0257 for COII and 0.0336 for $C y t b$ (Table S3).

The $C y t b$ sequence appears to be more effective and informative than other genes for the study of aphid population genetics.
However, due to a high level of nucleotide diversity, analysis of Cytb often led to ambiguous species identification $6.2 \%$ in the "best match" analysis and $14.5 \%$ in the "best close match" analysis). An example of the utility of Cytb occurred in the cluster comprising Cinara piniarmandicola Zhang, Zhang \& Zhong, C. bungeanae Zhang, Zhang \& Zhong and C. orientalis (Takahashi). C. piniarmandicola and $C$. orientalis cannot be distinguished from each other and were regarded as a whole. Using the COI and COII sequences, the two species formed a single cluster. In contrast, in the Cytb tree, they formed different clusters (Figure 3), as the maximum intra-specific divergence of $C$. piniarmandicola (0.0488) based on $C y t b$ gene is higher than the inter-specific divergence between C. piniarmandicola and C. bungeanae $(0.0222, \mathrm{SE}=0.0119)$.

\section{Comparison among the three mitochondrial regions}

In Lachninae, the absolute amount of intra-specific divergence among $C O I$ and $C y t b$ sequences was generally equal to or greater than that of the COII sequence. For example, in Lachnus, the 
Table 1. Analysis of intra- and interspecific divergence among congeneric species in selected genera of Lachninae.

\begin{tabular}{|c|c|c|c|c|}
\hline \multirow[b]{2}{*}{ Genera } & \multicolumn{4}{|c|}{ Analysis of intra- and inter-specific divergences of congeneric species in Lachninae } \\
\hline & $\begin{array}{l}\text { Average inter-specific } \\
\text { distance (COIICOIIICytb) }\end{array}$ & $\begin{array}{l}\text { Average intra-specific } \\
\text { distance (COIICOIIICytb) }\end{array}$ & Theta $(\theta)$ (cOl/ COIII Cytb) & $\begin{array}{l}\text { Coalescent depth (COIICOIII } \\
\text { Cytb) }\end{array}$ \\
\hline \multirow[t]{3}{*}{ Cinara Curtis } & $0.0875 \pm 0.0222$ & $0.0198 \pm 0.0185$ & $0.0225 \pm 0.0262$ & $0.0499 \pm 0.0414$ \\
\hline & $0.0981 \pm 0.0222$ & $0.0196 \pm 0.0202$ & $0.0157 \pm 0.0133$ & $0.0332 \pm 0.0296$ \\
\hline & $0.1039 \pm 0.0237$ & $0.0207 \pm 0.0224$ & $0.0162 \pm 0.0117$ & $0.0388 \pm 0.0320$ \\
\hline \multirow[t]{3}{*}{ Eulachnus del Guercio } & $0.0772 \pm 0.0090$ & $0.0074 \pm 0.0113$ & $0.0108 \pm 0.0162$ & $0.0125 \pm 0.0161$ \\
\hline & $0.0781 \pm 0.0130$ & $0.0064 \pm 0.0098$ & $0.0078 \pm 0.0141$ & $0.0087 \pm 0.0143$ \\
\hline & $0.0954 \pm 0.0144$ & $0.0083 \pm 0.0133$ & $0.0110 \pm 0.0197$ & $0.0123 \pm 0.0199$ \\
\hline \multirow[t]{3}{*}{ Lachnus Burmeister } & $0.0436 \pm 0.0369$ & $0.0094 \pm 0.0095$ & $0.0064 \pm 0.0003$ & $0.0202 \pm 0.0198$ \\
\hline & $0.0443 \pm 0.0370$ & $0.0065 \pm 0.0062$ & $0.0045 \pm 0.0028$ & $0.0099 \pm 0.0083$ \\
\hline & $0.0568 \pm 0.0521$ & $0.0098 \pm 0.0095$ & $0.0069 \pm 0.0055$ & $0.0129 \pm 0.0099$ \\
\hline \multirow[t]{3}{*}{ Stomaphis Walker } & $0.0867 \pm 0.0221$ & $0.0031 \pm 0.0042$ & $0.0017 \pm 0.0003$ & $0.0026 \pm 0.0045$ \\
\hline & $0.0820 \pm 0.0272$ & $0.0043 \pm 0.0058$ & $0.0024 \pm 0.0041$ & $0.0036 \pm 0.0062$ \\
\hline & $0.1192 \pm 0.0250$ & $0.0125 \pm 0.0000$ & $0.0125 \pm 0.0000$ & $0.0125 \pm 0.0000$ \\
\hline
\end{tabular}

doi:10.1371/journal.pone.0046190.t001

average intra-specific divergence of $C O I$ was $0.0094(\mathrm{SE}=0.0095)$ and of $C y t b$ was 0.0098 (SE $=0.0095)$; both were greater than the divergence of COII $(0.0065, \mathrm{SE}=0.0062)$. Though for some species the analysis of Cytb leads to ambiguous species identification, in other cases, it can provide useful information on population structure or on subtle differences within a species. An example of this can be found in Cinara tujafilina (del Guercio), the intra-specific Cytb divergence of which can reach 0.0843 .

The interspecific divergence of $C O I$ varies in different animal group. Among the 13,320 species pairs of animals, the divergence ranged from 0.00 to 0.54 , while most pairs of species $(79 \%)$ showed sequence divergence greater than 0.08 [2], and the mean sequence divergence among congeneric species was 0.18 for mayflies [22] and 0.164 for spiders [23]. In Lachninae, the interspecific divergence of $C O I$ was always less than the corresponding divergence of COII or Cytb (Tables 1, 2). For example, in Cinara, the average inter-specific divergence of $C O I$ among congeneric species was $0.0875(\mathrm{SE}=0.0222)$, which was lower than the divergence of COII (0.0981, $\mathrm{SE}=0.0222)$ and of Cytb $(0.1039$, $\mathrm{SE}=0.0237)($ Table 1). When we calculated the inter-specific divergence of species within the same tribe, $C O I$ likewise displayed the lowest divergence of the three markers (Table 2). In addition, Lachninae cannot form a monophyletic group in the COI tree, while in the COII and Cytb trees, the family is monophyletic (Figure 1). Under Hebert's criterion [2], using this sequence to identify a species would fail because the group is not monophyletic. Compared the efficiency of PCR amplification among three markers, COII was best and Cytb was worst. In addition, the COI

Table 2. Analysis of interspecific divergence of species within the tribes of Lachninae.

\begin{tabular}{llll}
\hline \multicolumn{4}{l}{ Analysis inter-specific divergences of species within con-tribe in Lachninae } \\
\cline { 2 - 4 } Tribes & COI & COII & Cytb \\
\hline Cinarini & $0.1039 \pm 0.0188$ & $0.1073 \pm 0.0137$ & $0.1142 \pm 0.0127$ \\
Lachnini & $0.1272 \pm 0.0179$ & $0.1274 \pm 0.0209$ & $0.1395 \pm 0.0225$ \\
\hline
\end{tabular}

gene yielded greater nonspecific amplification $(2.92 \%)$ than the other two markers.

Species were identified using the "best match" and "best close match" approaches. Analysis of the COII sequence yielded an identification frequency for the "best match" and "best close match" techniques of $96.9 \%$ and $90.8 \%$, respectively (Table 3), which was significantly higher than that for the other two markers. The frequency of successful identification using COI and $C y t b$ was similar for both approaches $(92.7 \%$ and $93.7 \%$ for the "best match" method and $83.9 \%$ and $83.7 \%$ for the "best close match" method, respectively). Thus, COII yielded the most accurate identification of Lachninae species in our study.

Our results suggest that $C O I I$ is a more reliable indicator of intra- and interspecies divergence than COI or Cytb. In addition, COII was efficiently amplified by PGR and provided accurate species identification. As species identification problems arose mainly in the case of indistinguishable sequences between species, a COII-based identification system should be effective in distinguishing Lachninae species from one another. However, at the level of both genus and tribe, there was significant overlap in all three markers. Similarly, none of the markers could easily distinguish morphologically similar species from each other (Figures 2, S2).

\section{A novel method to improve the DNA barcoding identification system}

The use of DNA barcoding as a method for rapid species identification and for the discovery of new groups requires that inter-specific variation exceeds intra-specific variation by one

doi:10.1371/journal.pone.0046190.t002 
Table 3. Successful identification of species based on "best match" and "best close match" analysis of the interspecific divergence of COI, COII and Cytb sequences among species within Lachninae.

\begin{tabular}{lll}
\hline Parameter & “Best match” & “Best close match” \\
\hline Success & $92.7 \% / 96.9 \% / 93.7 \%$ & $83.9 \% / 90.8 \% / 83.7 \%$ \\
Ambiguous & $2.7 \% / 3.1 \% / 6.3 \%$ & $11.0 \% / 8.0 \% / 14.5 \%$ \\
Misidentification & $4.6 \% / 0.0 \% / 0.0 \%$ & $5.1 \% / 1.2 \% / 1.8 \%$ \\
\hline doi:10.1371/journal.pone.0046190.t003 &
\end{tabular}

order of magnitude to establish a "barcoding gap" or the reciprocal monophyly of a species. In many cases, however, a "barcoding gap" is absent [24].

In our study, the "barcoding gap" was not present, and significant overlaps were formed in Lachninae using the COI, COII and Cytb markers. This overlap resulted in a high probability of ambiguous species identification or of species misidentification (Table 3). Therefore, we propose a new method to improve molecular species identification called "tag barcodes." In this approach, when a species is identified by DNA barcoding, the tag is added to the standard DNA barcode sequence. These tags can improve the efficiency of barcoding identification by removing the overlap between intra- and interspecific genetic divergence values.

This method is best illustrated using the following experimental data as an example. In traditional taxonomy, C. formosana (Takahashi) and C. pinea (Zetterstedt) are regarded as sister groups [25], and our molecular data support this view. For C. formosana and $C$. pinea, the mean intraspecific $C O I$ sequence divergence was 0.0165 and 0.0341 , and the maximum distance was 0.0476 and 0.0559 , respectively. A barcoding gap did not exist in these samples, with the overlapping regions ranging from 0.0506 to 0.0559 (Figure 4). Therefore, DNA barcoding would fail to identify samples that are in these regions.

"Tag barcodes" were used to resolve this problem. We first determined the composition of the tag for each species according to multiple alignments (Table 5). For example, for C. formosana (Takahashi), using the standard $658 \mathrm{bp}$ COI sequence as its DNA barcode, we attached the tag $(7,49,61,214,268,340,343,382$, $403,427,532)$ in the barcode; each number represents the standard barcode position of each highly variable site in sequence.

When we calculated the inter-specific divergence, if we retained the tag, the length of sequence is not change, so the inter-specific divergences are the same. However, if we deleted the tag when we calculated the intra-specific divergence, the length of sequences is shorter, and the intra-specific divergences are less than before. We believe that the highly variable sites have a negative impact on calculating intra-specific divergence, yet play an important role in distinguishing sequences among different species. This is due to the variable rate of evolution of certain loci in different species [26-28], which may interfere with the identification of individuals of the same species.

Following the application of tag barcodes, our results indicated that the average divergence between $C$. formosana and $C$. pinea was 0.0705 (ranging from 0.0506 to 0.0995 ) but that the average intraspecies divergence was less than previously calculated $(0.0086$ vs 0.0218$)$.

A barcoding gap of 0.0393-0.0506 existed in C. formosana (Takahashi) and C. pinea (Zetterstedt). Ideally, in a DNA barcoding analysis, the intraspecies divergence and interspecies divergence should be separated by a recognizable gap $[2,29]$ that functions to identify members of the same species. Therefore, with the use of "tag barcodes" that reduce the divergence overlap, DNA barcoding becomes a more efficient means of species identification.

To demonstrate the reliability of this improved method, we expanded our analysis to include a large number of Lachninae specimens. Prior to analysis, species in which the genetic divergence between congeneric species was less than 0.002 were removed; these species are likely synonymous. For the remaining species, we calculated the inter-specific and intra-specific divergence both before and after the application of tag barcodes. As a result of tag barcoding, the barcoding overlap in $\mathrm{COI}$ was nearly removed (Figure 5), and the rate of correct species identification by the "best close match" approach increased by up to $11.3 \%$ (from $83.9 \%$ to $95.2 \%$ ). Similar results were observed for COII and Cytb. Therefore, "tag barcodes" can improve the accuracy of identification by introducing a gap between inter- and intraspecific divergence values.

We recommend the following approach to identify species by DNA barcoding in taxa that contain many closely related species. (i) Get the standard DNA barcoding sequences for the unknown sample. In order to ensure the accuracy of identification, more than 10 individuals for testing are better. (ii) Compare these sequences to the DNA barcoding sequences of known species and determine which species are closely related to previously unknown samples. (iii) Compare the tag among these species to find the best species match. It is also possible to calculate the intra- and interspecific genetic divergence using "tag barcodes" to find the best species match.

Table 4. Morphological characteristics of Lachnus siniquercus Zhang, L. tropicalis (van der Goot), L. roboris (Linnaeus) and $L$. yunlongensis Zhang.

\begin{tabular}{|c|c|c|c|c|c|c|}
\hline \multirow[b]{2}{*}{ Species } & \multicolumn{6}{|c|}{ Morphological characteristics } \\
\hline & Body length (mm) & $\begin{array}{l}\text { Ratio of } \\
\text { antennal } \\
\text { (segment IV } \\
\text { and III) }\end{array}$ & $\begin{array}{l}\text { The number of } \\
\text { setae of } \\
\text { abdominal } \\
\text { tergite VIII }\end{array}$ & $\begin{array}{l}\text { Ratio of setae length of } \\
\text { antennal segment III and } \\
\text { widest diameter of the } \\
\text { segment }\end{array}$ & $\begin{array}{l}\text { Length ratio of } \\
\text { hind tibia and } \\
\text { body }\end{array}$ & $\begin{array}{l}\text { The number of } \\
\text { setae on cauda }\end{array}$ \\
\hline Lachnus siniquercus & 5.1 & 0.50 & $37-55$ & 0.50 & 0.73 & 60 \\
\hline Lachnus tropicalis & 3.1 & 0.33 & $18-25$ & 1.10 & 0.97 & $24-35$ \\
\hline Lachnus roboris & 4.4 & 0.41 & $48-49$ & 0.79 & 0.87 & $38-55$ \\
\hline Lachnus yunlongensis & 4.5 & 0.45 & $43-44$ & 0.67 & $0.82-0.87$ & $62-75$ \\
\hline
\end{tabular}




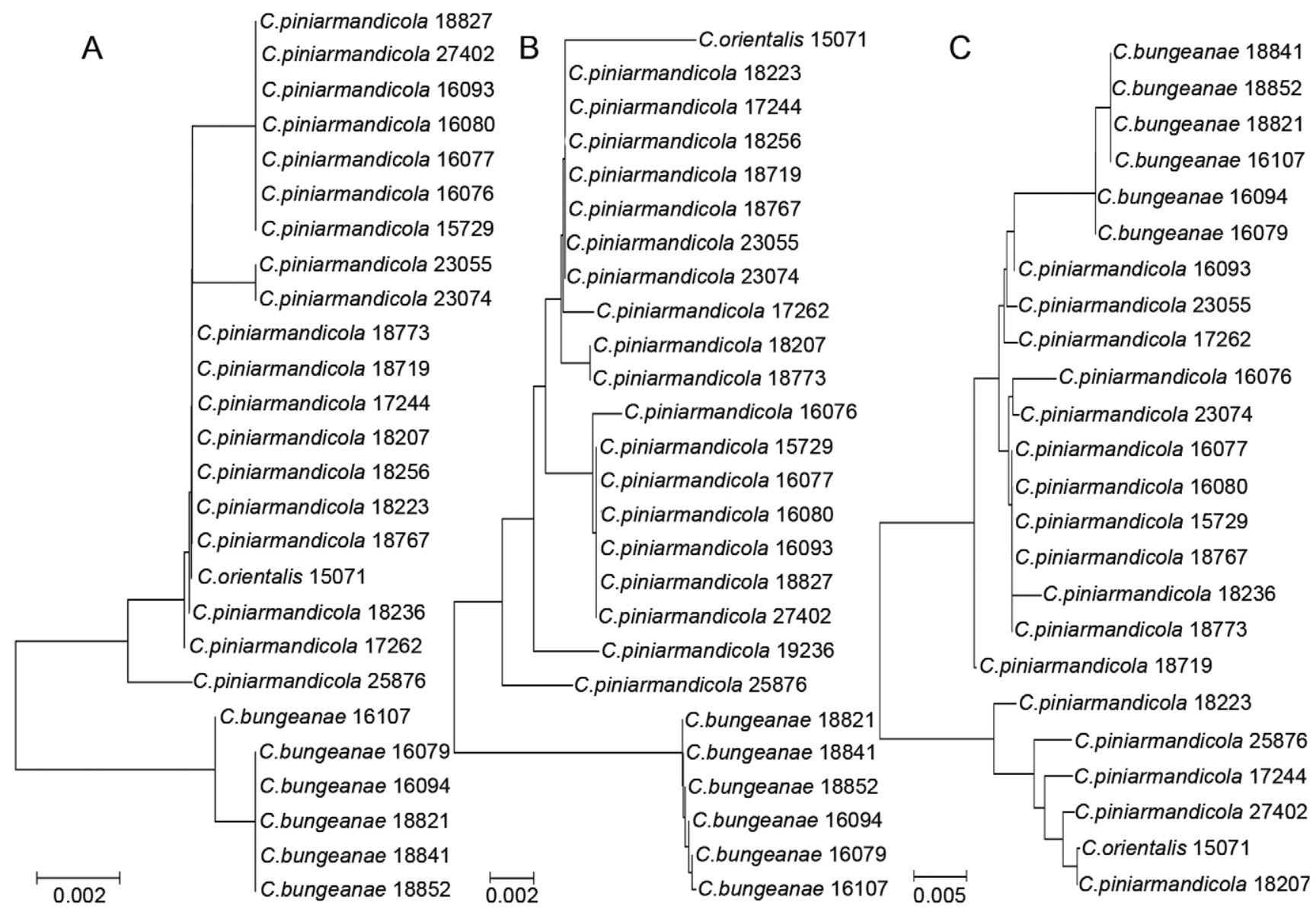

Figure 3. NJ analysis of K2P distances in Cinara piniarmandicola Zhang, Zhang \& Zhong, C. bungeanae Zhang, Zhang \& Zhong and C. orientalis (Takahashi). A, B, and C are the COI, COIl and Cytb NJ trees, respectively. doi:10.1371/journal.pone.0046190.g003

In summary, we suggest that specific markers suitable for different taxa should be chosen as standard DNA barcodes instead of using COI exclusively, which will improve the success rate of identification by DNA barcoding. By analyzing the aphid subfamily Lachninae in this study, we determined that the COII sequence yielded lower intra- and higher interspecific genetic divergence values for Lachninae compared to COI and Cytb. A COII-based identification system will therefore be more effective in identifying Lachninae species. Furthermore, the use of "tag barcodes" is a useful method through which the accuracy of identification by DNA barcoding can be improved by removing the overlap between intra- and interspecific genetic divergence. We recommended that this method be used in taxa containing many closely related species, and we suggest that this approach can be used to resolve the problem of species misidentification by conventional DNA barcoding.

\section{Materials and Methods}

Taxon sampling and data collection

Samples were selected to ensure the coverage of most Lachninae genera, including Cinara Curtis, Eulachnus del Guercio, Essigella del Guercio, Schizolachnus Mordvilko, Lachnus Burmeister, Longistigma Wilson, Maculolachnus Gaumont, Nippolachnus Matsumura, Pterochloroides Mordvilko, Pyrolachnus Basu et Hille Ris Lambers, Tuberolachnus Mordvilko, Stomaphis Walker, Protrama Baker and Trama von Heyden (Table S2). To avoid overestimating the sequence divergence between congeneric taxa due to the lack of a broad geographical sampling for a single species [4], we studied samples of selected species, including Cinara formosana (Takahashi), Cinara tujafilina (del Guercio), Cinara pinea (Mordvilko) and Lachnus tropicalis (van der Goot), which originated from geographically distant locations. The ingroup set included 1098 sequences representing 83 species, 14 genera and 3 tribes (Table S2). Among these sequences, we amplified 340 COI sequences, 354 COII sequences and 294 Cytb sequences and identified 110 sequences from European and North American samples that were downloaded from GenBank. The outgroups were Pineus armandicola (Zhang) (Adelgidae), Phylloxerina salicis (Lichtenstein) (Phylloxeridae), Aphis gossypii Glover (Aphidinae: Aphidini), Mindarus keteleerifoliae Zhang (Mindarinae) and Macrosiphoniella yomogifoliae (Shinji) (Aphidinae: Macrosiphini).

The collection information for each sample, including the location, the host plant and the collection date, is listed in Table S1. Specimens used for slide mounting were stored in $70 \%$ ethanol, while all other samples were stored in either $95 \%$ or $100 \%$ ethanol. All samples and voucher specimens were deposited in the National Zoological Museum of China, Institute of Zoology, Chinese Academy of Sciences, Beijing, China.

Each sample included several individual organisms. One to three individuals per sample were used for DNA isolation for molecular studies, and three to five individuals per sample were mounted on slides for morphological examination. Voucher 

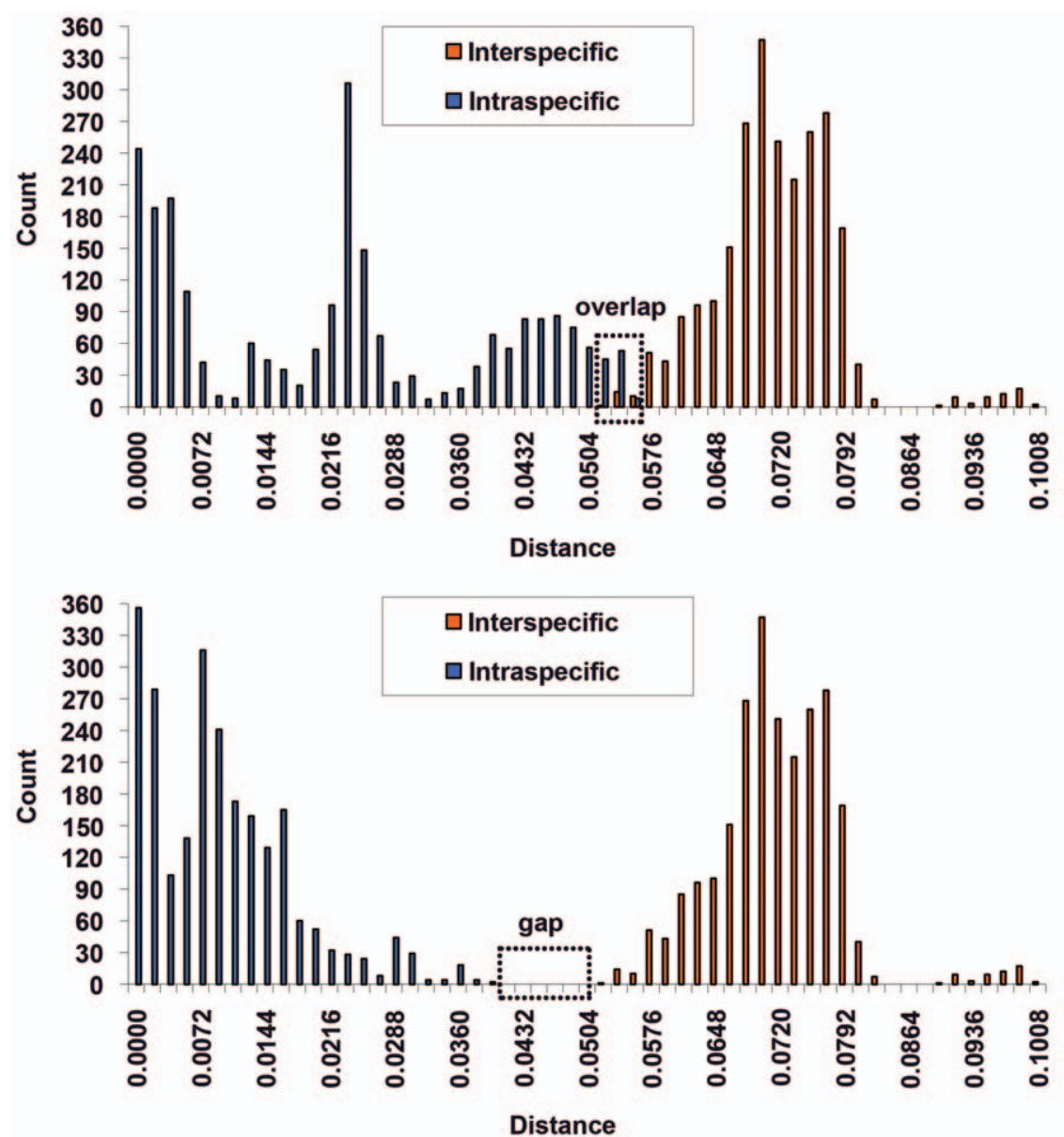

Figure 4. Frequency histogram of intra- and interspecific genetic divergence between C. formosana (Takahashi) and C. pinea (Zetterstedt) before (above) and after (below) using "tag barcodes." Genetic divergence was calculated using K2P model. doi:10.1371/journal.pone.0046190.g004

specimens from all samples were identified by their main morphological diagnostic features and were compared to previously identified specimens.

\section{DNA extraction, PCR and sequencing}

Total DNA was extracted from a single aphid preserved in $95 \%$ or $100 \%$ ethanol. Tissue homogenates were incubated at $55^{\circ} \mathrm{C}$ in lysis buffer (30 mM Tris-HCl [pH 8.0], $200 \mathrm{mM}$ EDTA, $50 \mathrm{mM}$ $\mathrm{NaCl}, 1 \%$ SDS and $100 \mu \mathrm{g} / \mathrm{ml}$ Proteinase K) for 5-7 hours, followed by a standard phenol-chloroform-isoamylalcohol (PCI) extraction with modifications [30]. DNA was precipitated from the supernatant with 2 volumes of cold ethanol, centrifuged, washed, dried and dissolved in 15-20 $\mu \mathrm{l}$ TE buffer and was stored at $4^{\circ} \mathrm{C}$ for later use.
The amplicon size of $C O I$ is approximately $660 \mathrm{bp}$; the primers used $\left(5^{\prime}-3^{\prime}\right)$ were ATTCAACGAATCATAAAGATATTGG and TAAACTTCTGGATGTCGAAAAAATCA [10]. The amplicon size of COII ranges from 668 to $671 \mathrm{bp}$; the primers used $\left(5^{\prime}-3^{\prime}\right)$ were CATTCATATTCAGAATTACG and GAGACGATTACTTGCTTTCAGTCATCT [31]. The amplicon size of Cytb ranges from 676 to $730 \mathrm{bp}$; the primers used $\left(5^{\prime}-3^{\prime}\right)$ were GATGATGAAATTTTGGATC and CTAATGGAATAACTCGTCG [32]. PCR amplification of COI was performed with an initial denaturation of $5 \mathrm{~min}$ at $94^{\circ} \mathrm{C}$ followed by 40 cycles under the following conditions: $94^{\circ} \mathrm{C}$ for $30 \mathrm{~s}, 50^{\circ} \mathrm{C}$ for $1 \mathrm{~min}$ and $72^{\circ} \mathrm{C}$ for $1 \mathrm{~min}$, with a final extension of $72^{\circ} \mathrm{C}$ for $10 \mathrm{~min}$. PCR for $\mathrm{COII}$ was performed with an initial denaturation of $3 \mathrm{~min}$ at $94^{\circ} \mathrm{C}$ followed by 35 cycles under the following conditions: $94^{\circ} \mathrm{C}$ for $30 \mathrm{~s}, 46^{\circ} \mathrm{C}$ for $1 \mathrm{~min}$ and $72^{\circ} \mathrm{C}$ for $1 \mathrm{~min}$, with a final

Table 5. The barcoding tags of two species, Cinara formosana (Takahashi) and Cinara pinea (Zetterstedt).

\begin{tabular}{ll}
\hline Species & Tag \\
\hline Cinara formosana (Takahashi) & $7,49,61,214,268,340,343,382,403,427,532$ \\
Cinara pinea (Zetterstedt) & $7,16,55,88,94,178,187,202,223,235,247,266,301,340,361,367,385,400,418,457,468,475,499$, \\
& $529,533,556,595,619$ \\
\hline
\end{tabular}

doi:10.1371/journal.pone.0046190.t005 

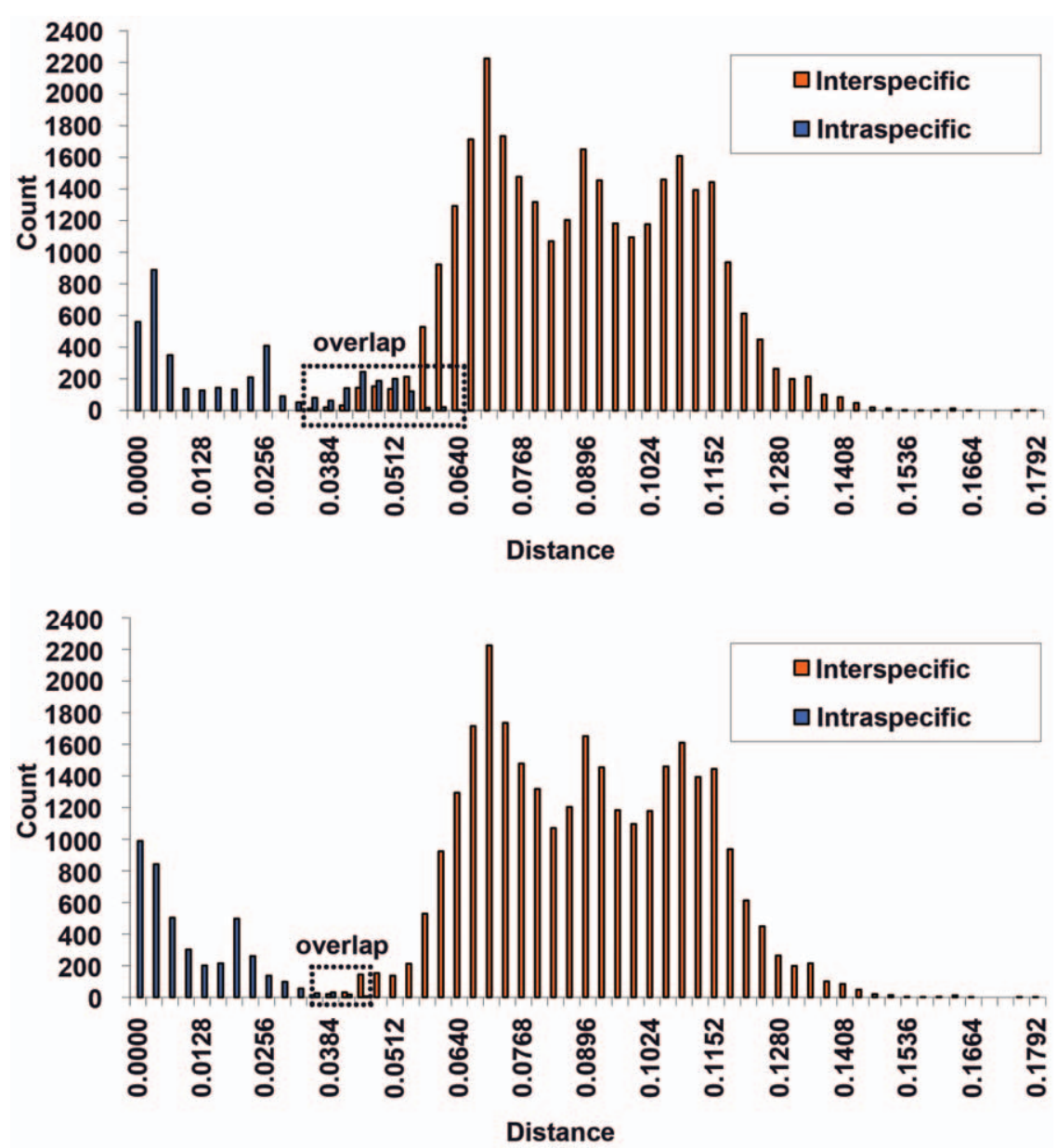

Figure 5. Frequency histogram of intra- and interspecific genetic divergence among Lachninae before (above) and after (below) using "tag barcodes." Genetic divergence was calculated using K2P model.

doi:10.1371/journal.pone.0046190.g005

extension of $72^{\circ} \mathrm{C}$ for $10 \mathrm{~min}$. PCR for Cytb was performed with an initial denaturation of $5 \mathrm{~min}$ at $95^{\circ} \mathrm{C}$ followed by 40 cycles under the following conditions: $95^{\circ} \mathrm{C}$ for $1 \mathrm{~min}, 48^{\circ} \mathrm{C}$ for $1 \mathrm{~min}$ $50 \mathrm{~s}$ and $72^{\circ} \mathrm{C}$ for $1 \mathrm{~min}$, with a final extension of $72^{\circ} \mathrm{C}$ for $10 \mathrm{~min}$.

Sequencing reactions were performed bidirectionally with the appropriate amplification primers using a BigDye Terminator Cycle Sequencing Kit v.2.0 (Applied Biosystems, USA) and an ABI 3730 automated sequencer (Applied Biosystems, USA). Each of the sequence reactions was repeated three times for reproducibility.

Chromatograms of the sense and antisense sequences were assembled and analyzed using Seqman software (DNASTAR, Inc.), and a consensus sequence was obtained. Multiple alignments were generated using CLUSTALX [33] and were subsequently pruned to a length of $657 \mathrm{bp}(C O I), 671 \mathrm{bp}(C O I I)$ or $730 \mathrm{bp}(C y t b)$. We confirmed that these sequences were correct by translating them in silico using Editseq software (DNASTAR, Inc. 1996). Sequences were deposited in GenBank, and accession numbers are provided in Table $\mathrm{S} 1$.

\section{Data analysis}

Nucleotide saturation was analyzed by plotting the number of transitions and transversions at each codon position against the Tamura \& Nei [34] (TN93) genetic distance using DAMBE [35].
Saturation was achieved if the mutation frequency leveled off as the sequence divergence increased. Intraspecific and interspecific (between congeneric species pairs) sequence divergence was based on Kimura' 2 parameter (K2P) distances for aphid species; divergence scores were calculated by MEGA 5.0. The K2P model provided the best metric when genetic distances were low [36]. Neighbor-joining (NJ) analysis [37] was used to examine relationships among taxa and population samples.

\section{Identifying species based on distance}

"Best match". We used TaxonDNA to identify the closest barcode match for each query. If both sequences were from the same species, the identification was considered successful, whereas mismatched sequences were counted as failures. Several equally best matches from different species were considered to be an ambiguous result.

"Best close match". We used TaxonDNA to plot the relative frequency of intraspecific distances to determine the threshold value below which $95 \%$ of all intraspecific distances were found. All sequences without a barcode match below this threshold value remained unidentified. For the remaining sequences, their identities were compared to the species identity of the closest barcode. If the species were identical, the identification was considered successful. The identification was considered to be a failure if the species identities were mismatched 
and was considered to be ambiguous if several equally best matches were found belonging to a minimum of two species.

\section{Attaching tags in the DNA barcode}

MEGA 5.0 was used for "tag barcodes" analysis. A multiple alignment of all studied barcode sequences from one species revealed highly variable sites that we termed "tags." The number in each tag represents the standard barcode position of each highly variable site in the sequence. Highly variable sites must vary by greater than $30 \%$ within a species. The more haplotypes are contained within a species, the more precise of tags can be found. This threshold value was derived from the statistical data obtained from Lachninae. If the threshold were lower (or too low), then there are too many tags representing a single species, and if the threshold were too high, then there may be too few tags to provide a unique identification for each species.

\section{Supporting Information}

Figure S1 Transitions ( $s$, in blue) and transversions ( $v$, in green) versus divergence for three mitochondrial gene sequences.

(JPG)

Figure S2 NJ analysis of K2P distances in Cinara pinea (Zetterstedt), . atrotibialis David \& Rajasingh and $C$.

\section{References}

1. McKenzie M, Chiotis M, Pinkert CA, Trounce IA (2003) Functional respiratory chain analyses in murid xenomitochondrial cybrids expose coevolutionary constraints of cytochrome b and nuclear subunits of complex III. Molecular Biology and Evolution 20: 1117-1124.

2. Hebert PDN, Cywinska A, Ball SL, de Waard JR (2003a) Biological identifications through DNA barcodes. Proceedings of the Royal Society of London Series B: Biological Sciences 270: 313-321.

3. Whitworth TL, Dawson RD, Magalon H, Baudry E (2007) DNA barcoding cannot reliably identify species of the blowfly genus Protocalliphora (Diptera: Calliphoridae). Proceedings of the Royal Society B: Biological Sciences 274: 1731-1739.

4. Meyer CP, Paulay G (2005) DNA barcoding: error rates based on comprehensive sampling. PLoS Biology 3: e422.

5. Cognato AI (2006) Standard percent DNA sequence difference for insects does not predict species boundaries. Journal of Economic Entomology 99: 10371045.

6. Song H, Buhay JE, Whiting MF, Crandall KA (2008) Many species in one: DNA barcoding overestimates the number of species when nuclear mitochondrial pseudogenes are coamplified. Proc Natl Acad Sci U S A 105: 13486-13491.

7. Chase MW, Salamin N, Wilkinson M, Dunwell JM, Kesanakurthi RP, et al. (2005) Land plants and DNA barcodes: short-term and long-term goals. Philosophical Transactions of the Royal Society B: Biological Sciences 360: 1889-1895.

8. Kress WJ, Wurdack KJ, Zimmer EA, Weigt LA, Janzen DH (2005) Use of DNA barcodes to identify flowering plants. Proc Natl Acad Sci U S A 102: 8369-8374.

9. Fazekas AJ, Burgess KS, Kesanakurti PR, Graham SW, Newmaster SG, et al. (2008) Multiple multilocus DNA barcodes from the plastid genome discriminate plant species equally well. PLoS One 3: e2802.

10. Foottit RG, Maw HEL, Von Dohlen CD, Hebert PDN (2008) Species identification of aphids (Insecta: Hemiptera: Aphididae) through DNA barcodes. Molecular Ecology Resources 8: 1189-1201.

11. Lee W, Kim H, Lim J, Choi HRAN, Kim Y, et al. (2010) Barcoding aphids (Hemiptera: Aphididae) of the Korean Peninsula: updating the global data set. Molecular Ecology Resources 11: 32-37.

12. Remaudière G, Remaudière M (1997) Catalogue des Aphididae du Monde. Institut National de la Recherche Agronomique, Paris. pp. 473.

13. von Dohlen CD, Rowe CA, Heie OE (2006) A test of morphological hypotheses for tribal and subtribal relationships of Aphidinae (Insecta: Hemiptera: Aphididae) using DNA sequences. Molecular Phylogenetics and Evolution 38: 316-329.

14. Heie OE (1980) The Aphidoidea (Hemiptera) of Fennoscandia and Denmark. I. General part. The families Mindaridae, Hormaphididae, Thelaxidae, Anoeciidae, and Pemphigidae. Fauna Entomol. Scand 9: 1-236.

15. Heie OE (1987) Palaeontology and phylogeny. In "Aphids: Their Biology, Natural Enemies, and Control". In: AK . Minks and P . Harrewijn, editors. Amsterdam: Elsevier. pp. 367-391. piniphila (Ratzeburg). $\mathrm{A}, \mathrm{B}$, and $\mathrm{C}$ are the $C O I$, COII and $C y t b$ NJ trees, respectively.

(TIF)

Table S1 The detailed collection information and GenBank accession numbers of Lachninae species included in this study.

(XLS)

Table S2 List of Lachninae species included in this study.

(DOCX)

Table S3 Mean intraspecific nucleotide divergence of Lachninae species generated using K2P model. (DOCX)

\section{Acknowledgments}

We gratefully thank all of the aphid collectors for their assistance, Ms. FenDi Yang for making slides of voucher specimens, and Prof. Fu-Min Lei for revising the early manuscript.

\section{Author Contributions}

Conceived and designed the experiments: G-XQ RC L-YJ. Performed the experiments: RC L-YJ. Analyzed the data: G-XQ RC L-YJ. Contributed reagents/materials/analysis tools: G-XQ L-YJ RC. Wrote the paper: RC G-XQ L-YJ.

16. Lahaye R, Van Der Bank M, Bogarin D, Warner J, Pupulin F, et al. (2008) DNA barcoding the floras of biodiversity hotspots. Proc Natl Acad Sci U S A 105: 2923-2928.

17. Zhang DX, Hewitt GM (1997) Assessment of the universality and utility of a set of conserved mitochondrial COI primers in insects. Insect Molecular Biology 6: $143-150$.

18. Zhang HC, Qiao GX (2006) Application of gene sequences in molecular phylogenetic study on Aphididae (Hemiptera). Acta Entomologica Sinica 49: 521-527.

19. Hebert PDN, Stoeckle MY, Zemlak TS, Francis CM (2004) Identification of birds through DNA barcodes. PLoS Biology 2: e312.

20. Hajibabaei M, Janzen DH, Burns JM, Hallwachs W, Hebert PD (2006) DNA barcodes distinguish species of tropical Lepidoptera. Proc Natl Acad Sci U S A 103: 968-971.

21. Ward RD, Zemlak TS, Innes BH, Last PR, Hebert PDN (2005) DNA barcoding Australia's fish species. Philosophical Transactions of the Royal Society B: Biological Sciences 360: 1847-1857.

22. Ball SL, Hebert PDN, Burian SK, Webb JM (2005) Biological identification of mayflies (Ephemeroptera) using DNA barcodes. Journal of the North American Benthological Society 24: 508-524.

23. Barrett RDH, Hebert PDN (2005) Identifying spiders through DNA barcodes. Canadian Journal of Zoology 83: 481-491.

24. Wiemers M, Fiedler K (2007) Does the DNA barcoding gap exist?-a case study in blue butterflies (Lepidoptera: Lycaenidae). Frontiers in Zoology 4: 16.

25. Zhang GX, Zhong TS (1983) Economic Insect Fauna of China. Fasc. 25. Homoptera, Aphidinae, part 1. Beijing: Science Press. pp.1-387

26. Philippe H, Lopez P (2001) On the conservation of protein sequences in evolution. Trends in Biochemical Sciences 26: 414-416.

27. Lopez P, Casane D, Philippe H (2002) Heterotachy, an important process of protein evolution. Molecular Biology and Evolution 19: 1-7.

28. Jeffroy O, Brinkmann H, Delsuc F, Philippe H (2006) Phylogenomics: the beginning of incongruence? TRENDS in Genetics 22: 225-231.

29. Hebert PDN, Ratnasingham S, de Waard JR (2003b) Barcoding animal life: cytochrome c oxidase subunit 1 divergences among closely related species. Proceedings of the Royal Society of London Series B: Biological Sciences 270: S96.

30. Sambrook J, Fritsch EF, Maniatis T (1989) Molecular Cloning: A Laboratory Manual. Cold Spring Harbour. New York: Coldspring Harbour Laboratory Press.

31. Stern DL (1994) A phylogenetic analysis of soldier evolution in the aphid family Hormaphididae. Proc Biol Sci 256: 203-209.

32. Harry M, Solignac M, Lachaise D (1998) Molecular evidence for parallel evolution of adaptive syndromes in fig-breeding Lissocephala (Drosophilidae). Mol Phylogenet Evol 9: 542-551.

33. Thompson JD, Gibson TJ, Plewniak F, Jeanmougin F, Higgins DG (1997) The CLUSTAL X windows interface: flexible strategies for multiple sequence alignment aided by quality analysis tools. Nucleic Acids Res 25: 4876-4882. 
34. Tamura K, Nei M (1993) Estimation of the number of nucleotide substitutions in the control region of mitochondrial DNA in humans and chimpanzees. Molecular Biology and Evolution 10: 512-526.

35. Xia X, Xie Z (2001) DAMBE: software package for data analysis in molecular biology and evolution. Journal of Heredity 92: 371-373
36. Nei M, Kumar S (2000) Molecular evolution and phylogenetics. New York: Oxford University Press.

37. Saitou N, Nei M (1987) The neighbor-joining method: a new method for reconstructing phylogenetic trees. Molecular Biology and Evolution 4: 406-425. 